\title{
Effect of Coil Dimensions on Dynamic Wireless Power Transfer for Electric Vehicles
}

\author{
Sahar Bareli ${ }^{1}$, Lidor Geri ${ }^{1}$, Yasha Nikulshin ${ }^{1}$, Oren Nahum ${ }^{23}$, Yuval Hadas ${ }^{2}$, Yosef Yeshurun ${ }^{1}$, Eyal Yaniv ${ }^{4}$, Shuki Wolfus ${ }^{1}$ \\ ${ }^{1}$ Dept. of Physics, Bar-Ilan University, Israel \\ ${ }^{2}$ Dept. of Management, Bar-Ilan University, Israel \\ ${ }^{3}$ Economics and Logistics Studies, Faculty of Economics, Ashkelon Academic College, Israel \\ ${ }^{4}$ Graduate School of Business Administration, Bar-Ilan University, Israel
}

\begin{abstract}
We explore the effects of various receiver coil dimensions and configurations on power transfer efficiency and cost of operation, using advanced simulation tools. We demonstrate that the spatial distribution of the magnetic field leads to a nonmonotonic dependence of the coupling coefficient on coil size. Thus, an optimal coil size, where the coupling coefficient peaks, should be regarded a crucial design parameter which affects the entire system performances. The incorporation of our findings into a multi-objective optimization algorithm is also discussed.
\end{abstract}

\section{Introduction}

Dynamic Wireless Power Transfer (DWPT) technology enables the process of charging the on board battery of an Electric (EV) while on the move along its travel path. Transmitter coils under the road transmit electromagnetic energy to receiver coils mounted under the car chassis. Since the process of charging the EV accompanies the car along its travel path, it allows the on-board battery to be minimized in dimensions, capacity and weight. The reduced battery carried by the EV leads to dramatic energy savings due to the reduced vehicle weight, as well as significant environmental advantages caused by minimizing $\mathrm{CO}_{2}$ emissions and other hazardous waste and contaminants [1]. In designing DWPT systems, one must consider receiver and transmitter coil dimensions, shapes, and alignments, as these factors drastically impact the magnetic flux collection efficiency of the system. As EVs become more and more popular, improving their charging efficiency and range becomes crucial for allowing the technology to further penetrate the transportation market. One method that allows for these improvements is the DWPT [2]-[5], in which the coils are electromagnetically coupled so that magnetic flux generated in the transmitting coil is only partially picked up by the receiving coils. One way to improve DWPT is to improve the electromagnetic coupling by varying the geometry, windings, and resonance parameters of the transmitting and/or the receiving coils. The coupling may also be improved by using magnetic materials. This paper explores the effects of varying the dimensions of the receiver and transmitter coils on the coupling coefficient, $\mathrm{k}$, which in turn, affects the overall efficiency of the power transfer. Considering physical restrictions such as vehicle dimensions, we show that $\mathrm{k}$ depends on the dimensions of the coil and its optimal value affects the design of the system.

Various types of coil configurations have been previously suggested for WPT (static wireless charging) and DWPT applications. These differ in shape, size, polarity (unipolar, bipolar) and use of magnetic cores. Some of these configurations were tested in, for example, the Korean OLEV project [6], [7]. Railing types were also explored in previous papers, such as the "I-type" module, which describes an "I" shaped power supply rail [8], which was implemented in one of the OLEV systems and offered good spatial magnetic flux coverage at a somewhat limited range. The S-type module was implemented in a newer OLEV system, which included an Sshaped power supply rail for better transmitter range [9]. This improved upon its predecessor by effectively doubling the distance between the receiver coils and the transmitter coils while also reducing EMF well below the guidelines. Unipolar and bipolar transmitter and receiver coils were also tested [3], [10]-[12]. While the unipolar coils are much easier to implement, as they require simple geometries, they are outperformed by their bipolar counterparts, since a bipolar configuration confines the flux lines into a more specific region under the receiver coils, increasing the coupling coefficient and reducing residual EM radiation. One of the most effective receiver bipolar configurations is the "doubleD" (DD) shape configuration, where two rectangular coils are laid side by side to form a "double-window"-like shape and are connected in opposite direction to pick the net sum of two 
opposing sign magnetic field lines. Still, the DD configuration is very susceptible to misalignments. Clearly, when the EV travels along the transmitter coils track, large variations in the picked-up flux occur, including a point of minimal signal, where the DD layout is positioned above one of the transmitter coils. The double-D quadrature (DDQ) configuration [3] includes an additional single coil mounted at the center of the DD layout. It provides a good solution to DWPT, since it can pick up increased amounts of magnetic flux lines relative to, its similar in size, DD configuration. It is also far less susceptible to misalignments, as the Q coil covers the "dead" zones of the DD coils, effectively increasing the flux pickup. While the DDQ configuration is undoubtedly effective, there are still many optimization opportunities to explore - namely the dimensions of the receiver DD and Q coils and the distance separating between each of the D coils. This paper explores the effect of the dimension variations of the receiver and transmitter coils in the DDQ configuration on the electromagnetic coupling and energy transfer efficiency. We have found that substantial improvement in energy transfer can be obtained by such modifications to the classic DDQ configuration. This work demonstrates that $\mathrm{k}$ increases with increasing coil size, reaching a maximum and then decreases with further increase in coil size. This result is analyzed via magnetic field distribution simulations and explained by the amount of net flux penetrating the receiver coils. Practically, the results of the analysis show that for a pre-set air gap distance between transmitter and receiver coils, the receiver size can be used as a design parameter for obtaining optimal energy efficiency.

\section{Simulation Method}

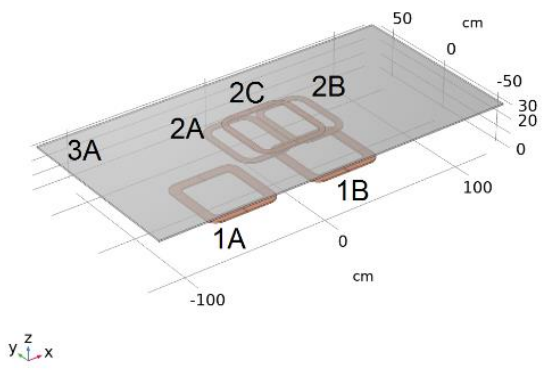

Figure 1: Initial layout of the simulated coil array. 1A, 1B: transmitter coils. 2A, 2B: DD receiver coils. 2C: Q shaped receiver coil on top of the DD receiver coils. 3A: Ferromagnetic ferrite plate.

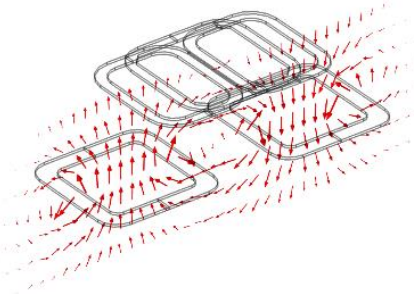

Figure 2: Flux lines generated in the bipolar transmitter coils.

Electromagnetic simulations of DWPT coil configurations have been performed using COMSOL Multiphysics. As a starting point to our simulations, we used a DDQ configuration shown in figure 1. Each transmitter coil was defined to have 11 turns, while the receiver coils have 3.5 turns each. The transmitter coils also form a DD configuration and are placed $25 \mathrm{~cm}$ apart. The transmitter coils are connected in series in a "figure of eight" manner, such that the current in one coil flows clockwise, while the current in the second coil flows counterclockwise. The receiver coils are connected in a similar manner, so that flux in opposite directions crossing each ' $\mathrm{D}$ ' coil is integrated and contributes to the induced voltage. The transmitter coils therefore create a bipolar flux spatial distribution as seen in figure 2, where the flux which enters one coil exits through the other. In all simulations for the transmitter coils, a 50 A RMS, $85 \mathrm{kHz}$ sinewave current was generated, using frequency domain simulation. On top of the receiver coil array, a ferrite plate has been placed, which serves to divert flux lines into the receiver coils, as well as a magnetic shield for reducing electromagnetic non-ionizing radiation level at passengers' level to well below the allowed standards [13]. Variations to the receiver and transmitter coil dimensions have been made, specifically stretching the "radius" of the DD coils to cover more of the EV's length or width, yielding more effective rectangular shapes. These changes were made with the goal of collecting more flux lines at the receiving coils, and were proven to be very successful, as described in the next section.

\section{Results and Discussion}

The electromagnetic coupling coefficient of two inductors is given by:

$$
k=\frac{L_{1,2}}{\sqrt{L_{1} L_{2}}}
$$

where $L_{1}$ and $L_{2}$ are the self-inductances of the transmitter and receiver coils, respectively. The self-inductances are directly correlated to the geometric properties of the coils. $L_{1,2}$ is defined to be the mutual inductance between the receiver and transmitter coils:

$$
L_{1,2}=V_{2} / \frac{d i_{1}}{d t}
$$


where $\boldsymbol{V}_{\mathbf{2}}$ is the voltage across the receiver coils, and $\boldsymbol{i}_{\mathbf{1}}$ is the current in the transmitter coils.

The first set of simulations explores configurations similar to the base configuration seen in figure 1 , but with varying receiver coils dimensions. In each simulation step, both length and width of the receiver coils were either stretched or compressed by the same value, yielding a Q-shaped geometry in each step. These simulations were run multiple times, each time with a different transmitter coils diameter, the results of which can be seen in figure 3 . The voltage induced in the receiver coils increases with the increse in transmitter coil size. Also, for each transmitter size, there is a corresponding receiver size that receives a maximal amount of voltage.

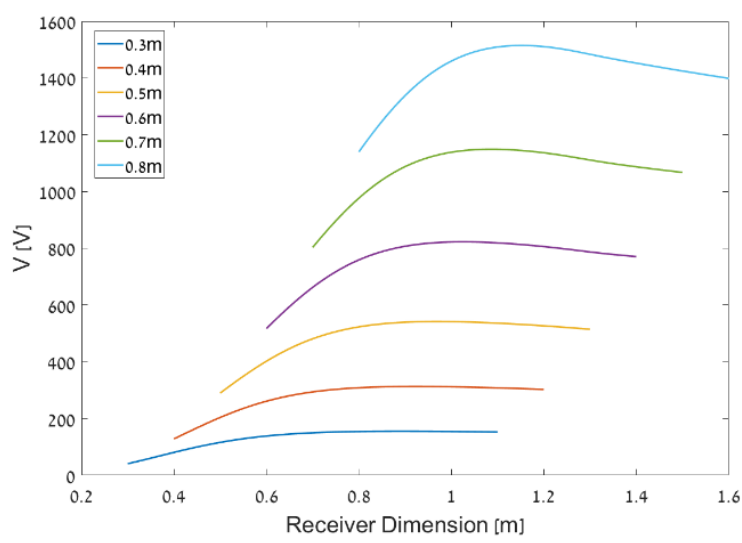

Figure 3: Voltage across receiver as a function of the change in $D D$ coil dimensions. Different curves represent different transmitter coil dimensions from 0.3 to $0.8 \mathrm{~m}$.

In practice, the vehicle width has a tighter dimension restriction than its length. Hence, in the second set of simulations, coil width was fixed at $0.5 \mathrm{~m}$ and only the length of the receiver coils was varied. The results are displayed in figure 4. In this case, the effect is slightly less dramatic than the 2-directions change, yet still substantial. Voltage curves reach a maximum at around the same points as in the previous case and decrease in a weaker way thereafter.

Figure 5 displays the coupling coefficient, $\mathrm{k}$, as a function of dimension changes. The self and mutual inductances of equations 1, 2 were calculated from the voltage induced in each coil by varying the current in the appropriate coil. Clearly, $\mathrm{k}$ increases with the increase of the transmitter coils dimensions. For transmitting coils of $0.3 \mathrm{~m}$ length, $\mathrm{k}$ is less than 0.1 and increases up to $\sim 0.23$ for $0.8 \mathrm{~m}$ long coils. The optimal operation point is now clearer and corresponds to the peak in $\mathrm{k}$. For any selected transmitter coil size, the coupling between the coils improves with the increase in the receiver coil length up to a maximum. Further increase in length results in decreasing $\mathrm{k}$ hence, reduced energy transfer efficiency.

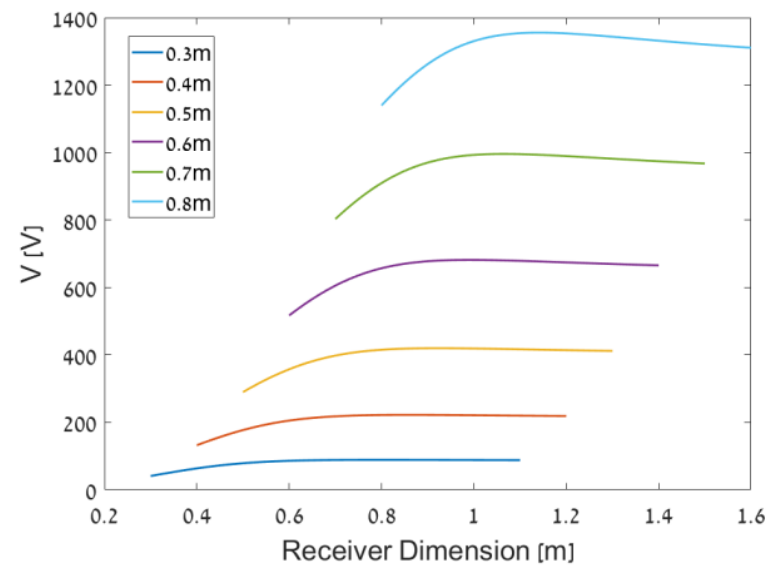

Figure 4: Length dependent receiver voltage. Different curves represent different transmitter coil dimensions from 0.3 to $0.8 \mathrm{~m}$.

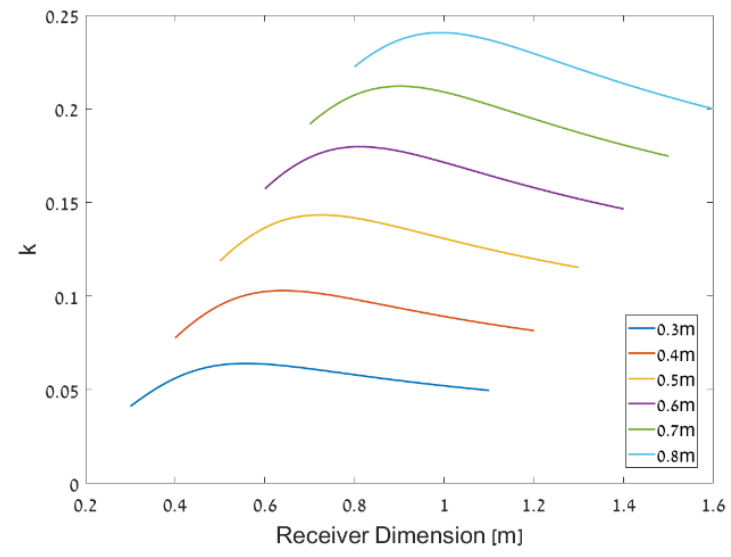

Figure 5: Coupling coefficient as a function receiver coil length. Different curves represent different transmitter coil dimension from 0.3 to $0.8 \mathrm{~m}$.

The increase in $\mathrm{k}$ with increasing transmitter coil size is straight forward. Since the coupling is proportional to the ratio $\mathrm{r} / \mathrm{h}$, ( $\mathrm{h}$ being the distance between coils and $\mathrm{r}$ its typical dimension) [2], increasing the size of the transmitter coils is equivalent to reducing the distance between the transmitter and receiver coils. Therefore, it is desirable to have as large as possible transmitting coils and the limit is set by cost of the conductor, the road trenching and the need to keep the dimension smaller than the EV width to minimize stray fields in its vicinity. To explain non monotonic behavior of $\mathrm{k}$ for each selected transmitter size, one must look at the magnetic field distribution in the XY and XZ planes of Fig.1. Figure 6 describes the $\mathrm{Z}$ component of the magnetic field $\left(B_{Z}\right)$ in the receiver coils $X Y$ plane, for the case of $0.5 \mathrm{~m}$ sized transmitters. $B_{z}$ reaches its absolute value maximum at about $\mathrm{x}=38 \mathrm{~cm} /-38 \mathrm{~cm}$ respectively. These points represent the maximum amount of flux that can be "caught" by the receiver coils. Once we venture past these points, $B_{z}$ starts diminishing. 
Once we reach a negative field, (at around $90 \mathrm{~cm}$ ), the induced voltage on each receiver declines.

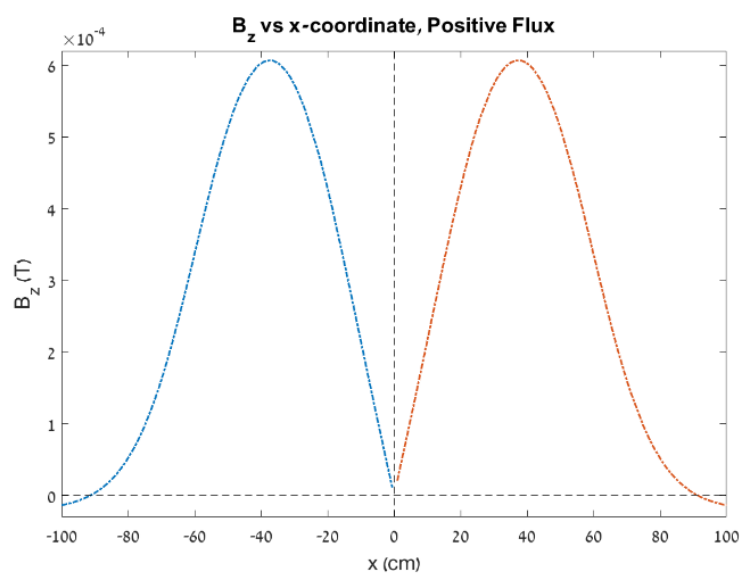

Figure 6: Position dependent $B_{z}$ along the x-axis. The direction of the flux displayed in this figure represents the flux that is seen by

each reciever. Maximas are observed at around $x= \pm 38 \mathrm{~cm}$.

Figure 7 represents the integral of $B_{z}$ integrated over the entire $\mathrm{x}$ axis. This correlates well with figures 5 and 6 , as the overall flux starts plateauing rapidly at around $0.7 \mathrm{~m}$. This is the point where the flux in figure 6 decreases rapidly and is about to flip and become negative, therefore obstructing further flux collection. Also, in figure 5 we see that $\mathrm{k}$ starts decreasing rapidly after that point which strengthens the claim that once we go past this point, flux collection becomes less significant. Figures 6 and 7 also show that the flux collection plateaus around zero, meaning it can be potentially more effective to separate the DD configuration and add a gap between the two "D" coils. Figure 8 describes the changes to $\mathrm{k}$ in different separation cases for the case of the default $38 \mathrm{~cm}$ 'D' "radius" case. Each line represents a different distance between the Dshaped coils. We can therefore gather that a separation of 5$10 \mathrm{~cm}$ is ideal for this case, as it reaches the highest efficiency of all other tested cases, while also being more cost efficient than some of the alternatives, as it is generally smaller in size. Finally, figure 9 shows the total energy transfer efficiency in the $10 \mathrm{~cm}$ separation case, with the default $50 \mathrm{~cm}$ transmitters. The efficiency is maximal at the same point where $\mathrm{k}$ is maximal. Further increasing the size of the receiver slightly decreases the efficiency.

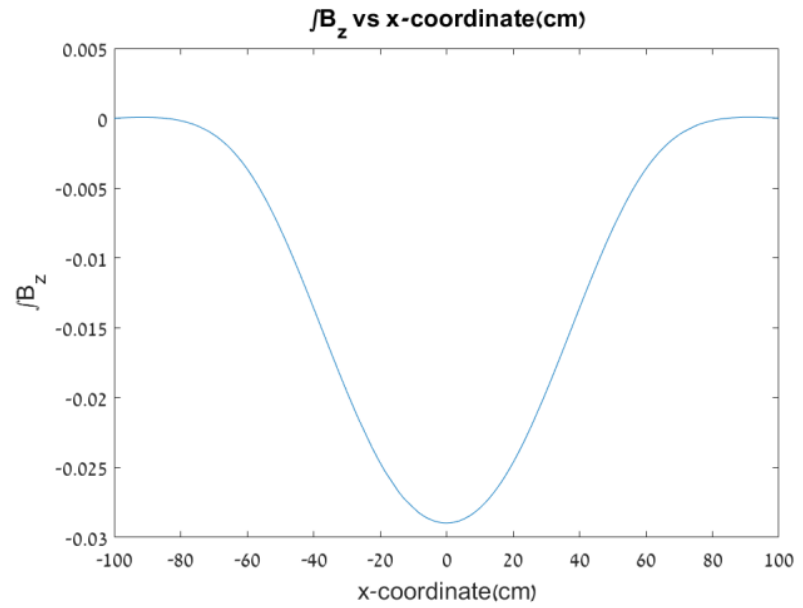

Figure 7: Integrated $z$ component of the magnetic flux density by the $x$-axis.

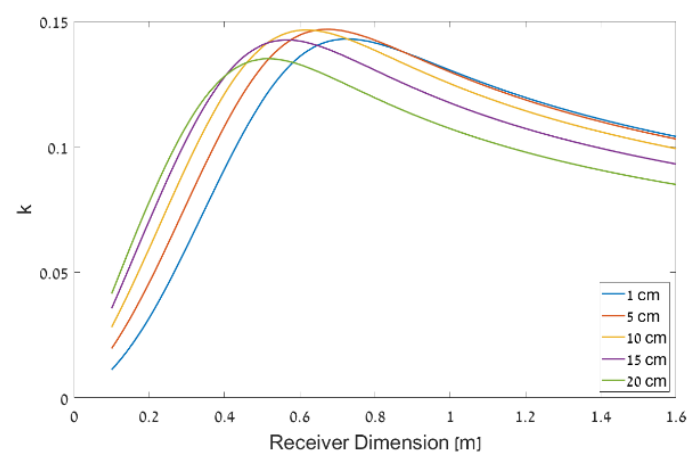

Figure 8: Comparision between different seperating gaps.

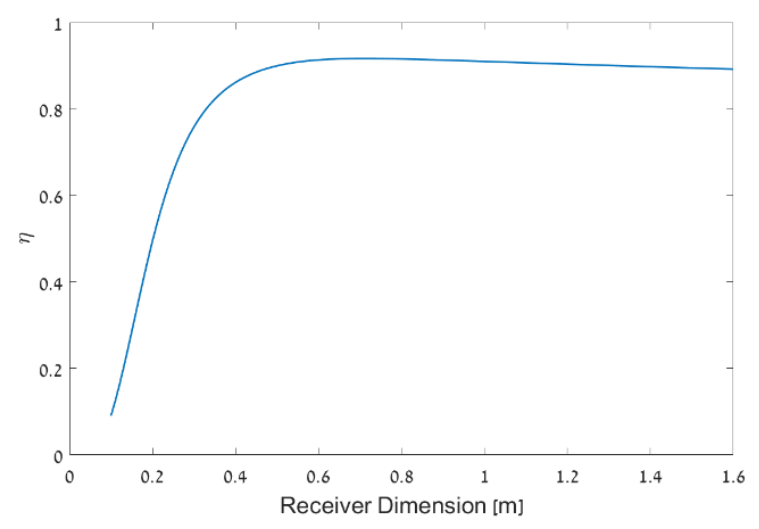

Figure 9: Energy transfer efficiency of the $10 \mathrm{~cm}$ separation case.

\section{Multi-objective Optimization}

As previously mentioned, DWPT has advantages; Charging the EV dynamically (in-motion) can be translated to a significantly reduced battery size requirement, and the fact that the charging process may occur under both static and 
dynamic conditions, hence offering extended or even unlimited travel range. For example, the BYD K9 electric bus has a $324 \mathrm{kWh}$ battery weighing $1500 \mathrm{~kg}$ which requires recharge of 6 hours every $250 \mathrm{~km}$, compared to an the OLEV bus that uses a $13 \mathrm{kWh}$ battery weighing only $130 \mathrm{~kg}$ that can be charged in less than 5 minutes [14]. As most public transport buses have fixed routes, it is possible to design an optimal allocation of charging stations along the route, considering the dwell time at the stops. Several papers have dealt with the problem of finding the optimal location for installing transmitting coils from a logistical managerial point of view [15]-[18]. A recent paper by Nahum and Hadas [14] proposed an optimal allocation model for designing a systemwide network of wireless bus charging stations. In this work, the suggested approach consisted of a multi-objective model that selected the locations for the charging stations (i.e., road embedded transmitting coils) while (a) minimizing the costs, (b) maximizing the environmental benefit, and (c) minimizing the number of charging stations.

As an example, the multi-objective optimization model proposed by Nahum and Hadas [14] was adapted to examine the deployment of infrastructure in the shuttle route of BarIlan University (a single route), with 17 bus stops. Based on dwell times, energy consumption profile and costs of batteries and charging stations, an optimal solution with a $1 \mathrm{kWh}$ battery priced at $\$ 1500$, and nine charging station, was found.

However, as described in the current study, the position of transmitter coils is not the only factor that influences flux collection efficiency. Other transmitter and receiver coil parameters (dimensions, shape, alignments and location) influence energy transfer efficiency as well and should be taken into consideration when designing a system-wide network of wireless bus charging stations. Specifically, by considering the variations in battery sizes as a consequence of the increased coupling between the receivers and transmitters, the coil parameters can be integrated in such multi-objective optimization algorithm. Based on the work of Redelbach et. Al. [19], an estimated $150 \mathrm{~J}$ per $\mathrm{kg}$ and $\mathrm{km}$ drive was used to include the effect of battery reduction due to coupling improvement in a multi-objective optimization analysis of the Bar-Ilan in-campus shuttle service. It was found that in such cases of high coupling, wireless charging in specific shuttle stations would suffice to energize the entire shuttle service. These results will be described in a separate publication.

\section{Conclusions}

We have shown how various changes to the classic DDQ configuration for the receivers can greatly affect the coupling coefficient between the receiver and transmitter systems, which results in better and more efficient energy transfer. In particular, it was found that an optimal size of receiver coil exists for every selected size of transmitter coil; The coupling coefficient increases with increasing receiver size, peaks at this optimal size and decreases thereafter. These findings were interpreted by analyzing the spatial magnetic field distribution, which have shown that the net magnetic flux which crosses the receiver coils start to decrease above the optimal dimension due to magnetic field direction reversal. Our findings can be implemented in multi-objective optimization processed and be used to design better DWPT coil layouts which decrease battery sizes in EVs, save capital and operating costs and reduce $\mathrm{CO} 2$ emissions.

\section{Acknowledgements}

The research was funded in part by the Israeli Ministry of Environmental Protection. S. Bareli acknowledges the scholarship by the Israeli Smart Transportation Research Center (ISTRC). The authors thank O. Ezer and H. Rumbak with Electreon Ltd. for their information sharing and useful discussions. 


\section{References}

[1] O. Shimizu, S. Nagai, T. Fujita, and H. Fujimoto, "Potential for CO2 Reduction by Dynamic Wireless Power Transfer for Passenger Vehicles in Japan," Energies, vol. 13, no. 13, p. 3342, 2020.

[2] Y. Gao, A. Ginart, K. B. Farley, and Z. T. H. Tse, "Misalignment effect on efficiency of wireless power transfer for electric vehicles," in 2016 IEEE Applied Power Electronics Conference and Exposition (APEC), 2016, pp. 3526-3528.

[3] C. C. Mi, G. Buja, S. Y. Choi, and C. T. Rim, "Modern advances in wireless power transfer systems for roadway powered electric vehicles," IEEE Trans. Ind. Electron., vol. 63, no. 10, pp. 6533-6545, 2016.

[4] S. Y. Choi, B. W. Gu, S. Y. Jeong, and C. T. Rim, "Advances in wireless power transfer systems for roadway-powered electric vehicles," IEEE J. Emerg. Sel. Top. power Electron., vol. 3, no. 1, pp. 18-36, 2014.

[5] Z. Zhang, H. Pang, A. Georgiadis, and C. Cecati, "Wireless power transfer-An overview," IEEE Trans. Ind. Electron., vol. 66, no. 2, pp. 1044-1058, 2018.

[6] S. Lee, J. Huh, C. Park, N.-S. Choi, G.-H. Cho, and C.-T. Rim, "On-line electric vehicle using inductive power transfer system," in 2010 IEEE Energy Conversion Congress and Exposition, 2010, pp. 1598-1601.

[7] Y. D. Ko and Y. J. Jang, "The optimal system design of the online electric vehicle utilizing wireless power transmission technology," IEEE Trans. Intell. Transp. Syst., vol. 14, no. 3, pp. 1255-1265, 2013.

[8] J. Huh, S. W. Lee, W. Y. Lee, G. H. Cho, and C. T. Rim, "Narrow-width inductive power transfer system for online electrical vehicles," IEEE Trans. Power Electron., vol. 26, no. 12, pp. 3666-3679, 2011.

[9] S. Y. Choi, S. Y. Jeong, B. W. Gu, G. C. Lim, and C. T. Rim, "Ultraslim S-type power supply rails for roadwaypowered electric vehicles," IEEE Trans. Power Electron., vol. 30, no. 11, pp. 6456-6468, 2015.

[10] G. A. Covic, J. T. Boys, M. L. G. Kissin, and H. G. Lu, “A three-phase inductive power transfer system for roadwaypowered vehicles," IEEE Trans. Ind. Electron., vol. 54, no. 6, pp. 3370-3378, 2007.

[11] G. A. Covic and J. T. Boys, "Inductive power transfer," Proc. IEEE, vol. 101, no. 6, pp. 1276-1289, 2013.

[12] M. Budhia, G. Covic, and J. Boys, "Magnetic design of a three-phase inductive power transfer system for roadway powered electric vehicles," in 2010 IEEE Vehicle Power and Propulsion Conference, 2010, pp. 1-6.

[13] J. Lin et al., "ICNIRP Guidelines for limiting exposure to time-varying electric and magnetic fields $(1 \mathrm{~Hz}$ to 100 kHz).," Health Phys., vol. 99, pp. 818-836, 2010.

[14] O. E. Nahum and Y. Hadas, "Multi-objective optimal allocation of wireless bus charging stations considering costs and the environmental impact," Sustainability, vol. 12, no. 6, p. 2318, 2020.

[15] Y. J. Jang, E. S. Suh, and J. W. Kim, "System architecture and mathematical models of electric transit bus system utilizing wireless power transfer technology," IEEE Syst. J., vol. 10, no. 2, pp. 495-506, 2015.

[16] Y. Liu, X. Weng, J. Wan, X. Yue, H. Song, and A. V Vasilakos, "Exploring data validity in transportation systems for smart cities," IEEE Commun. Mag., vol. 55, no. 5, pp. 26-33, 2017.

[17] M. Xylia, S. Leduc, P. Patrizio, F. Kraxner, and S. Silveira,
"Locating charging infrastructure for electric buses in Stockholm," Transp. Res. Part C Emerg. Technol., vol. 78, pp. 183-200, 2017.

[18] M. Xylia, S. Leduc, P. Patrizio, S. Silveira, and F. Kraxner, "Developing a dynamic optimization model for electric bus charging infrastructure," Transp. Res. Procedia, vol. 27, pp. 776-783, 2017.

[19] M. Redelbach, M. Klötzke, and H. E. Friedrich, "Impact of lightweight design on energy consumption and cost effectiveness of alternative powertrain concepts," 2012. 